BU-HEPP-08-16

CASPER-08-06

\title{
A Non-Standard String Embedding of $E_{8}$
}

\author{
Richard Obousy, ${ }^{*}$ Matthew Robinson, ${ }^{\dagger}$ and Gerald B. Cleaver ${ }^{\ddagger}$ \\ Center for Astrophysics, Space Physics \& Engineering Research \\ Department of Physics, Baylor University, Waco, TX 76798-7316
}

\begin{abstract}
An algorithm to systematically and efficiently generate free fermionic heterotic string models was recently introduced [1]. This algorithm has been adopted by the Free Fermionic Model Construction (FFMC) program at Baylor University. As its first application, the algorithm is being applied to systematically generate the complete set of free fermionic heterotic string models with untwisted left-moving (worldsheet supersymmetric) sectors, up to continually advancing Layer and Order. Statistical analysis of this study will be reported in the near future. However, in a series of separate notes we will be reporting some of the more interesting models that appear along the way. In this, our first such note, we reveal a different string embedding of $E_{8}$ than is standard. That is, rather than realize $E_{8}$ via an $S O(16)$ embedding, $\mathbf{2 4 8}=\mathbf{1 2 0}+\mathbf{1 2 8}$, we realize it via an $S U(9)$ embedding, $\mathbf{2 4 8}=\mathbf{8 0}+\mathbf{8 4}+\overline{\mathbf{8 4}}$. This is obtained in a Layer 1, Order 6 model for which modular invariance itself dictates a gravitino sector accompany the gauge sector.
\end{abstract}

*Richard_K_Obousy@baylor.edu

${ }^{\dagger}$ M_Robinson@baylor.edu

${ }^{\ddagger}$ Gerald_Cleaver@baylor.edu 


\section{Systematic Investigation of Free Fermionic Heterotic String Models}

An algorithm [1] to systematically and efficiently generate free fermionic [2, 3] heterotic models was recently introduced. As a first application, we have initiated an indepth study of the statistics of the gauge groups in free fermionic heterotic strings with only untwisted left-moving (worldsheet supersymmetric) sectors. Our approach enables a complete study of all gauge group models to be generated and analyzed with extreme efficiency, up to continually increasing Layers (the number of gauge basis vectors) and Orders (the lowest positive integer $N$ that transforms, by multiplication, each basis vector back into the untwisted sector $\bmod (2))$. In this initial study the models have either $\mathcal{N}=4$ or $\mathcal{N}=0$ spacetime SUSY, depending on whether the gravitino sector is or is not present, respectively. The primary goal of our research is to systematically improve the understanding of the statistical properties and characteristics of free fermionic heterotic models, a process that is underway by a collection of research groups $[4,5]$. However, as particularly interesting models appear in the course of our program, we will separately report on such models. The first of these models appears at Layer 1, Order 6 and requires a graviton sector. The intersting feature of this model is that it provides an alternative embedding of $E_{8}$, based not on the $E_{8}$ maximal subgroup $S O(16)$, but on $E_{8}$ 's alternate maximal subgroup $S U(9)$.

\section{Review of $E_{8}$ String Models in 4 and 10 Dimension}

The $S O(16)$ realization of $E_{8}$ is well known: We start with the uncompactified $D=10, \mathcal{N}=1$ SUSY $S O(32)$ heterotic string in light-cone gauge. Free fermion construction generates this model from two basis boundary vectors: the ever-present all-periodic vector, $\mathbf{1}$, and the supersymmetry generating vector $\mathbf{S}$ [2]:

$$
\begin{aligned}
& \mathbf{1}=\left[(1)^{8} \|(1)^{32}\right] \\
& \mathbf{S}=\left[(1)^{8} \|(0)^{32}\right] .
\end{aligned}
$$

The 496 (adjoint) rep of $S O(32)$ is produced by the untwisted boundary vector $\mathbf{0}=\mathbf{1}+\mathbf{1}$,

$$
\mathbf{0}=\left[(0)^{8} \|(0)^{32}\right]
$$

To transform the uncompactified $D=10, \mathcal{N}=1$ SUSY $S O(32)$ heterotic model into the $D=10, \mathcal{N}=1$ SUSY $E_{8} \otimes E_{8}$ model, all that is required is the additional twisted basis boundary vector [2],

$$
\mathbf{I}^{O}=\left[(0)^{8} \|(1)^{16}(0)^{16}\right] .
$$


The GSO projection of $\mathbf{I}^{O}$ onto $\mathbf{0}$ reduces the untwisted sector gauge group to $S O(16)_{O} \otimes S O(16)_{H}$ by reducing its massless gauge states to the adjoint reps $\mathbf{1 2 0}_{\mathbf{O}} \otimes \mathbf{1}$

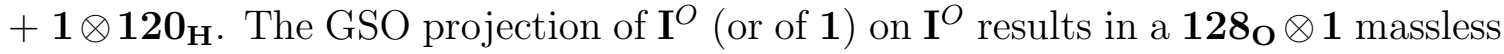
spinor rep of definite chirality. Further, the GSO projection of $\mathbf{1}$ onto

$$
\mathbf{I}^{H}=\mathbf{I}^{O}+\mathbf{1}+\mathbf{S}=\left[(0)^{8} \|(0)^{16}(1)^{16}\right]
$$

produces a massless spinor rep $\mathbf{1} \otimes \mathbf{1 2 8}_{\mathbf{H}}$ of $S O(16)_{H}$ with matching chirality.

Thus, the boundary sectors $\mathbf{0}$ and $\mathbf{I}^{O}$ produce the $\mathbf{2 4 8}$ (adjoint) of an observable $E_{8}$ via the $S O(16)$ embedding

$$
248=120+128
$$

while the boundary sectors $\mathbf{0}$ and $\mathbf{I}^{H}$ produce the same for a hidden sector $E_{8}^{H}$

When the $E_{8} \otimes E_{8}$ model is compactified to four dimensions, without any twist applied to the compact dimensions, the basis vectors become,

$$
\begin{aligned}
\mathbf{1} & =\left[(1)^{2},(1,1,1)^{6} \|(1)^{44}\right] \\
\mathbf{S} & =\left[(1)^{2},(1,0,0)^{6} \|(0)^{44}\right] \\
\mathbf{I}^{O} & =\left[(0)^{2},(0,0,0)^{6} \|(1)^{16}(0)^{28}\right] .
\end{aligned}
$$

Because

$$
\mathbf{I}^{H}=\mathbf{I}^{O}+\mathbf{1}+\mathbf{S}=\left[(0)^{2},(0,1,1)^{6} \|(0)^{16}(1)^{28}\right],
$$

is no longer a massless sector, the gauge group is $E_{8}^{O} \otimes S O(22)$ (with $\mathcal{N}=4$ SUSY). An additional massless twisted sector,

$$
\mathbf{I}^{H^{\prime}}=\left[(0)^{0},(0,0,0)^{6 \mid} \mid(0)^{16},(1)^{16},(0)^{6}\right],
$$

is required to reclaim the second $E_{8}{ }^{*}$

\section{$3 \quad E_{8}$ from $S U(9)$}

Our systematic research of free fermionic gauge models, revealed at Layer 1, Order 3 (more precisely Layer 1 , Order $6=\operatorname{Order}(2) \times \operatorname{Order}(3))$ as explained below) an intersting alternative realization of $E_{8}$ The simplest possible massless gauge sector for Order 3 is

$$
\mathbf{I}^{3}=\left[(0)^{0},(0,0,0)^{6 \mid} \mid\left(\frac{2}{3}\right)^{18},(0)^{26}\right]
$$

The non-integer values in $\mathbf{I}^{3}$ produce a GSO projection on the untwisted sector that breaks $S O(44)$ down to $S U(9) \otimes U(1) \otimes S O(26)$. The charges of the $S U(9)$ non-zero

${ }^{*}$ In this note we we do not discuss the gauge group of the left-moving sector, since it belongs to the $N=4$ gravity multiplet and disappears for $N<2$. 
roots are of the form $\pm\left(1_{i},-1_{j}\right)$ for $i$ and $j \neq i$ denoting one of the first 9 rightmoving complex fermion. Combined with the zero roots of the Cartan Subalgrabra, these form the $\mathbf{8 0}$ (adjoint) rep of $S U(9)$ The $U(1)=\sum i=1{ }^{9} U(1)_{i}$ charge is $\operatorname{Tr} Q_{i}$. The $S O(26)$ generators have the standard charges of $\pm\left(1_{r}, \pm 1_{s}\right)$ with $r$ and $s \neq r$ denoting one of the last 13 right-moving complex fermion.

However, two of the the modular invariance requirements for basis vectors $\mathbf{V}_{i}$ and $\mathbf{V}_{j}[2]$, specifically

$$
\begin{aligned}
N_{i, j} \mathbf{V}_{i} \cdot \mathbf{V}_{j} & =0(\bmod 4), \text { and } \\
N_{i} \mathbf{V}_{i} \cdot \mathbf{V}_{i} & =0(\bmod 8),
\end{aligned}
$$

necessitate that $\mathbf{I}^{3}$ be expressed as a spacetime fermion, rather than spacetime boson. That is, the required basis boundary vector to produce a gauge factor of $S U(9)$ in the untwisted sector in like manner to (3.1) is

$$
\mathbf{I}^{6}=\left[(1)^{0},(1,0,0)^{6} \mid\left(\frac{2}{3}\right)^{18},(0)^{26}\right] .
$$

As an Order $6=$ Order 2 x Order 3, basis boundary vector, (3.4) satisfies $(3.2,3.3)$. $2 \mathbf{I}^{6}=\mathbf{I}^{3}$ is then a massless gauge sector, as is $4 \mathbf{I}^{6}=-\mathbf{I}^{3}$. Note also that $3 \mathbf{I}^{6}$ is the gravitino sector $S$. Hence $\mathbf{S}$ need not, and cannot, be a separate basis vector.

The GSO projections of $\mathbf{1}$ and $\mathbf{I}^{6}$ on $I^{3}$ and $-I^{3}$ yield massless gauge states from two sets of charges. Charges in the first set have the form

$$
\pm\left(-\frac{2}{3}{ }_{i_{1}},-\frac{2}{3}{ }_{i_{2}},-\frac{2}{3}{ }_{i_{3}}, \frac{1}{3}{ }_{i_{4}}, \frac{1}{3}{ }_{i_{5}}, \frac{1}{3}{ }_{i_{6}}, \frac{1}{3}{ }_{i_{7}}, \frac{1}{3} i_{8}, \frac{1}{3}{ }_{i_{9}}\right),
$$

with all subscripts different and each denoting one of the first 9 complex fermions. States in $I^{3}$ and $-I^{3}$ vary by their overall charge sign and form the $\mathbf{8 4}$ and $\overline{\mathbf{8 4}}$ reps of $S U(9)$, respectively Thus, together the sectors $\mathbf{0}, I^{3}$, and $-I^{3}$ contain the $\mathbf{8 0}, \mathbf{8 4}$ and $\overline{\mathbf{8 4}}$ reps of $S U(9)$, from which $\mathbf{2 4 8}=\mathbf{8 0}+\mathbf{8 4}+\overline{\mathbf{8 4}}$ emerges. Thus, here $E_{8}$ is obtained from its second maximal subgroup $S U(9)$.

The second set of charges are of the form,

$$
\pm\left(\frac{1}{3}, \frac{1}{3}, \frac{1}{3}, \frac{1}{3}, \frac{1}{3}, \frac{1}{3}, \frac{1}{3}, \frac{1}{3}, \frac{1}{3}, \pm 1_{r}\right),
$$

with $1_{r}$ denoting a unit charge of one of the 13 complex fermions generating the $S O(26)$ Cartan subalgebra. Hence, the charges in this set are orthogonal to $E_{8}$, but have non-zero dot products with $U(1)=\sum i=1{ }^{9} U(1)_{i}$ charged states, and unit dot products with the $S O(26)$ generators. Thus, this second set of states enhance $S O(26)$ to $S O(28)$. The complete gauge group is thus $E_{8} \otimes S O(28)$. Since the gravitino sector is a multiple of $I^{6}$, the model has inherent $\mathcal{N}=4$ SUSY.

The whole process can be followed again with the addition of another basis boundary vector $\mathbf{I}^{6 H}$ isomorphic with $\mathbf{I}^{6}$, but that has no non-zero right-moving charges in common with $\mathbf{I}^{6}$ :

$$
\mathbf{I}^{6 H}=\left[(1)^{0},(1,0,0)^{6} \mid(0)^{18},\left(\frac{2}{3}\right)^{18},(0)^{8}\right] .
$$


$\mathbf{I}^{6 H}$ will produce a second $E_{8}$ from a parallel $S U(9)$ embedding. The $S O(8)$ of the untwisted sector would be enhanced by both of the $U(1)$ 's associated with the two $S U(9)$ 's to $S O(12)$, giving a standard $E_{8} \otimes E_{8} \otimes S O(12)$ model, but with an $S U(9) \times$ $S U(9)$ embedding for $E_{8} \otimes E_{8}$.

Heterotic models have an $S O(44)$ rotational redundancy in their charge expressions (which we are taking into account in our statistical analysis). In terms of solely the gauge sectors, our $E_{8}$ embedding from $S U(9)$ can be understood as a specific $S O(18) \in S O(44)$ rotation of the initial charge lattice. In the $S O(16)$ basis, a set of simple roots for $E_{8}$ are

$$
\begin{aligned}
& E_{1}=(+1,-1,0,0,0,0,0,0) \\
& E_{2}=(0,+1,-1,0,0,0,0,0) \\
& E_{3}=(0,0,+1,-1,0,0,0,0) \\
& E_{4}=(0,0,0,+1,-1,0,0,0) \\
& E_{5}=(0,0,0,0,+1,-1,0,0) \\
& E_{6}=(0,0,0,0,0,+1,-1,0) \\
& E_{7}=(0,0,0,0,0,+1,+1,0) \\
& E_{8}=\left(-\frac{1}{2},-\frac{1}{2},-\frac{1}{2},-\frac{1}{2},-\frac{1}{2},-\frac{1}{2},-\frac{1}{2},-\frac{1}{2}\right),
\end{aligned}
$$

where we choose a positive chirality $\mathbf{1 2 8}$ spinor. For an $S O(18)$ rotation we need 9 charge states, so we we will add an zero charge onto the $E_{8}$ charges and include a $U(1)$ generator with defining charge generator

$$
U_{9}=(0,0,0,0,0,0,0,0,1) .
$$

Alternately, a simple set of roots for the $S U(9)$ basis is

$$
\begin{aligned}
& E_{1}^{\prime}=(+1,-1,0,0,0,0,0,0,0) \\
& E_{2}^{\prime}=(0,+1,-1,0,0,0,0,0,0) \\
& E_{3}^{\prime}=(0,0,+1,-1,0,0,0,0,0) \\
& E_{4}^{\prime}=(0,0,0,+1,-1,0,0,0,0) \\
& E_{5}^{\prime}=(0,0,0,0,+1,-1,0,0,0) \\
& E_{6}^{\prime}=(0,0,0,0,0,+1,-1,0,0) \\
& E_{7}^{\prime}=(0,0,0,0,0,0,+1,0,-1) \\
& E_{8}^{\prime}=\left(-\frac{1}{3},-\frac{1}{3},-\frac{1}{3},-\frac{1}{3},-\frac{1}{3}, \frac{2}{3}, \frac{2}{3},-\frac{1}{3}, \frac{2}{3}\right) .
\end{aligned}
$$

In the $S U(9$ basis, there is also an additonal $U(1)$ of the form

$$
U_{9}^{\prime}=\left(\frac{1}{3}, \frac{1}{3}, \frac{1}{3}, \frac{1}{3}, \frac{1}{3}, \frac{1}{3}, \frac{1}{3}, \frac{1}{3}, \frac{1}{3}\right)
$$


The $S O(16)$ embedding of $E_{8}$ can be transformed into the $S U(9)$ embedding of $E_{8}$ via a Weyl rotation that yields

$$
\begin{aligned}
E_{7}^{\prime} & =\frac{1}{2}\left(E_{7}-E_{6}\right)-U_{9} \\
E_{8}^{\prime} & =\frac{2}{3}\left(E_{8}+U_{9}\right) \\
U_{9}^{\prime} & =\frac{2}{3}\left(-E_{8}+\frac{1}{2} U_{9}\right) .
\end{aligned}
$$

Note also that the rotation between these $E_{8}$ embeddings can be expressed in terms of partition function equivalences involving Theta-function product identities [6].

\section{Summary}

In this note we presented an alternative embedding for $E_{8}$, involving not its maximal subgroup $S O(16)$, rather its alternate maximal subgroup $S U(9)$. Instead of the $\mathbf{2 4 8}$ (adjount) rep of $E_{8}$ generated as $\mathbf{2 4 8}=\mathbf{1 2 0}+\mathbf{1 2 8}$ of $S O(16)$, we constructed a $D=4$ model in which it is generated as $\mathbf{2 4 8}=\mathbf{8 0}+\mathbf{8 4}+\overline{\mathbf{8 4}}$ of $S U(9)$. Interestingly, we found that in this model that modular invariance requires the basis boundary vector responsibble for the pair of massless gauge sectors that yields the $\mathbf{8 4}+\overline{\mathbf{8 4}}$ reps to also produce the gravitino-producing sector. The model starts out with $\mathcal{N}=4$ SUSY. Thus, this alternate $E_{8}$ embedding cannot occur in a model without either broken or unbroken SUSY (i.e., a model that lacks a gaugino sector).

\section{Acknowledgments}

Research funding leading to this manuscript was partially provided by Baylor URC grant 0301533BP. 


\section{References}

[1] M. Robinson, G. Cleaver, and M. Hunziker, Free Fermionic Heterotic Model Building and Root Spaces, BU-HEPP-08-03, CASPER-08-05, arXiv:0810.5094 [hep-th].

[2] I. Antoniadis, C. Bachas, and C. Kounnas, Nucl. Phys. B289 (1987) 87; H. Kawai, D.C. Lewellen, and S.H.-H. Tye, Nucl. Phys. B288 (1987) 1; I. Antoniadis and C. Bachas, Nucl. Phys. B298 (1988) 586; H. Kawai, D.C. Lewellen, J.A. Schwartz, and S.H.-H. Tye, Nucl. Phys. B299 (1989) 431

[3] H. Dreiner, J. Lopez, D.V. Nanopoulos, and D. Reiss, Nucl. Phys. B320 (1989) 401; G. Cleaver, Nucl. Phys. B456 (1995) 219.

[4] A.E. Faraggi, C. Kounnas, S.E.M. Nooij, and J. Rizos, [hep-th/0311058]; Nucl. Phys. B695 (2004) 41; R. Donagi and A.E. Faraggi, Nucl. Phys. B694 (2004) 187; A.E. Faraggi, S. Forste, and C. Timirgaziu, JHEP 0608 (2006) 057; A.E. Faraggi, C. Kounnas, and J. Rizos, Phys. Lett. B648 (2007) 84; Nucl. Phys. B744 (2007) 208; Nucl. Phys. B799 (2008) 19; G. Cleaver, A.E. Faraggi, E. Manno, and C. Timirgaziu, Phys. Rev. D78 (2008) 046009; T. Catelin-Jullien, A.E. Faraggi, C. Kounnas, and J. Rizos, arXiv:0807.4084 [hep-th]; A.E. Faraggi, arXiv:0809.2641 [hep-th].

[5] K. Dienes, Phys. Rev. D73 (2006) 106010; K. Dienes and M. Lennek, Phys. Rev. D75 (2007) 026008; AIP Conf. Proc.903 (2007) 505; arXiv:0809.0036 [hep-th]; K. Dienes, M. Lennek, D. Senechal, and V. Wasniik, Phys. Rev. D75 (2007) 126005; New J. Phys. 10 (2008) 085003.

[6] D. Mumford, Tata Lectures on Theta I, (Birkhauser, Boston, 1983). 